\title{
Short-Term Effect of Botulinum Toxin A Injection on Spastic Equinovarus Foot in Cerebral Palsy Patients: A Study Using the Foot Pressure Measurement System
}

\author{
Su Min Son, $\mathrm{MD}, \mathrm{PhD}^{1}$, In Silk Park, $\mathrm{MSc}^{2}$, Jin Sun Yoo, $\mathrm{MD}^{1}$ \\ ${ }^{1}$ Department of Physical Medicine and Rehabilitation, Yeungnam University College of Medicine, Daegu; \\ ${ }^{2}$ Korean Pedorthic Institute, Goyang, Korea
}

Objective To evaluate the therapeutic effect of botulinum toxin A (BTX-A) injection on spastic gastrocnemius (GCM) and tibialis posterior muscles (TPo) by using the foot pressure measurement system (FPMS).

Methods Eighteen ambulatory CP patients were recruited in this study. BTX-A was injected into the GCM at a dose of 6-12 units/ $\mathrm{kg}$ and TPo at a dose of 4-9 units/kg according to the severity of equinus and varus deformity. Foot contact pattern, pressure time integral (PTI), coronal index using the FPMS and Modified Ashworth Scale (MAS), and visual inspection of gait pattern were used for evaluation of the therapeutic effect of BTX-A injection. Clinical and FPMS data were statistically analyzed according to the muscle group.

Results A significant decrease in the MAS score of the GCM and TPo was observed, and spastic equinovarus pattern during gait showed improvement after injection. The GCM+TPo injection group showed a significant decrease in forefoot, lateral forefoot pad, and lateral column PTI, and a significant increase in hindfoot PTI and coronal index. In the GCM only injection group, forefoot PTI and lateral column PTI were significantly decreased and hindfoot PTI was significantly increased. The TPo only injection group showed a significant decrease in lateral column PTI and a significant increase in the coronal index. Change in PTI in the hindfoot showed a significant correlation with the change in MAS score of the GCM. Change in PTI of the lateral column and coronal index showed a significant correlation with the change in MAS score of the TPo.

Conclusion The FPMS demonstrated the quantitative therapeutic effect of BTX-A on abnormal pressure distribution in equinovarus foot in detail. The FPMS can be a useful additional tool for evaluation of the effect of BTX-A injection.

Keywords Equinovarus foot, Type A botulinum toxins, Foot pressure, Foot scan, Cerebral palsy 


\section{INTRODUCTION}

Equinovarus foot is the most common musculoskeletal complication in children with cerebral palsy (CP) $[1,2]$. The equinus foot is related to spasticity or contracture of the gastrocnemius muscle (GCM), and the varus foot is related to spasticity or contracture of the tibialis posterior muscle (TPo) $[3,4]$. While walking with the equinovarus foot, abnormal initial contact with the lateral border of the forefoot produces an inappropriate base of weight support; thus, limiting functional ambulation and independent activities of daily living $[5,6]$.

Various treatments, including physical therapy, bracing, casting, nerve block, and surgical lengthening of the gastrocnemius-soleus complex have been used in management of the equinovarus foot $[1,3,7,8]$. Among these therapeutic modalities, botulinum toxin A (BTX-A) has been widely used due to its safety and effectiveness. BTX-A enables reduction in spasticity of muscles and improvement of gait pattern by denervation of spastic muscles [9]. With regard to BTX-A injection, MAS and gait pattern assessment by visual inspection or gait analysis have been considered for determining the optimum target muscle or optimum dose. Weight-bearing pattern and foot pressure during gait cycle are also important factors for BTX-A injection. However, there is a limitation of assessing weight-bearing pattern or foot pressure using MAS or visual inspection.

The foot pressure measurement system (FPMS) is known to be useful in providing quantitative information on weight bearing pattern of the foot during the gait cycle [10]. Several studies have used the FPMS to investigate the therapeutic effect of patients with rheumatoid arthritis, diabetes mellitus, and several forefoot problems [1118]. Using the FPMS, detailed quantitative values, including those in the forefoot, midfoot, hindfoot, and medial and lateral foot sections, can be obtained; this information enables clinicians to make a more precise diagnosis regarding foot deformity and to perform a quantitative assessment of the therapeutic effect. In addition, this measurement tool can be easily applied to younger patients with cerebral palsy who are often uncooperative $[1,2,19-23]$.

In this study, we used the FPMS to investigate the therapeutic effect of BTX-A injection in patients with equinovarus foot due to spastic GCM or TPo.

\section{MATERIALS AND METHODS}

\section{Subjects}

Eighteen patients were recruited and selected on the basis of the following inclusion criteria by two expert physiatrists: 1) spastic hemiplegic or diplegic CP;2) equinovarus deformity of the foot, which was defined by ankle plantar flexion lower than $0^{\circ}$ and initial contact with the 5th metatarsal head during gait cycle [24];3) ambulatory patients diagnosed as having Gross Motor Function Classification System (GMFCS) level I or II; 4) no history of orthopedic surgery of the lower extremities or selective dorsal rhizotomy; 5) no history of BTX-A injection within the last four months; 6) no definite dyskinetic movement such as athetosis or dystonia; 7) absence of definite contracture of the lower extremity; 8) no definite contraindication for BTX-A injection; and 9) volunteers whose parents had applied on their behalf to participate in this study. Among the 18 participants (22 limbs; 15 males and three females; median age, 8.00 years; range, 2-17 years), 14 patients had hemiplegic $\mathrm{CP}$ and the other four patients had diplegic CP. As a result, 22 limbs of 18 subjects were assessed. The study was approved by the Institutional Review Board of a Yeungnam University College of Medicine and written informed consent was obtained from the parents of all participants.

\section{Clinical evaluation}

Assessment of the Modified Ashworth Scale (MAS) score and visual inspection of gait pattern were performed before and after BTX-A injection in all patients. The MAS is a widely used measurement tool, and it is scored as follows: 0 , no increase in muscle tone; 1 , slight increase in muscle tone at the end of the range of motion; 2, slight increase in muscle tone through less than half of the range of motion; 3 , more marked increase in muscle tone through most of the range of motion; 4, considerable increase in muscle tone; and 5, joint is rigid. Physical examinations and gait pattern assessment were performed by an expert physiatrist. MAS score of the GCM was evaluated in the supine position with maximal extension of the knee during maximal ankle dorsiflexion from maximal ankle plantar flexion [25]. MAS score of the soleus was evaluated in the prone position with $90^{\circ}$ flexion of the knee during maximal ankle dorsiflexion from maximal ankle plantar flexion [25]. MAS score of the 
TPo was evaluated in the prone position with $90^{\circ}$ flexion of the knee during maximal ankle dorsiflexion, eversion, and pronation from maximal ankle plantar flexion, inversion, and supination to assess the degree of varus [25].

\section{Foot pressure measurement system}

The FPMS (TPScan; BioMechanics, Goyang, Korea) was used for evaluation of dynamic foot pressure. Patients were instructed to walk naturally at a self-selected speed and look straight ahead. Before the test, patients practiced walking for nearly $2 \mathrm{~m}$ in order to become adapted to the FPMS. Dynamic foot pressure data during walking were recorded on the FPMS floor mat $\left(40.5 \times 40.5 \mathrm{~cm}^{2}\right)$ at the middle of the ambulation test. Subjects underwent five walking tests in order to obtain valid results. The parameters obtained from the FPMS were divided into two categories, including the foot contact pattern and the pressure time integral (PTI). For the foot contact pattern, contact length and contact width of the forefoot, midfoot, and hindfoot were measured by two investigators according to maximal length of contact length, forefoot, midfoot, and hindfoot in visualized results from the FPMS (Fig. 1). Dynamic foot pressure results for each section were automatically calculated by the FPMS. Results from PTI data were also provided as automatically divided sec-

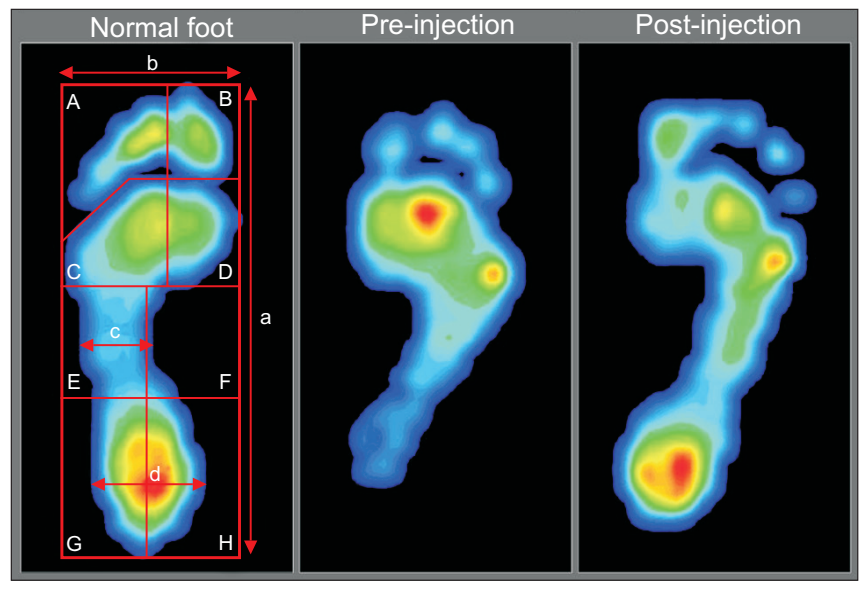

Fig. 1. Foot pressure measurement image. The foot pressure image of a normal foot and the changed image of equinovarus foot pressure between pre-injection and post-injection in a single child (A, toe; $\mathrm{B}$, hallux; $\mathrm{C}$, lateral forefoot; D, medial forefoot; E, lateral midfoot; F, medial midfoot; $\mathrm{G}+\mathrm{H}$, hind foot; $\mathrm{a}$, contact length; $\mathrm{b}$, forefoot contact width; c, midfoot contact width; and d, hindfoot contact width). tions-toe, forefoot pad, arch, and heel. Each section was automatically subdivided as follows: the toe section was subdivided into hallux ('B'), second toe, and third, fourth, and fifth toes ('A'); the forefoot section was subdivided into medial forefoot pad ('D') and lateral forefoot pad (' $C$ '); the arch section was subdivided into medial arch ('F') and lateral arch ('E') and the heel section was subdivided into medial heel ('H') and lateral heel ('G') (Fig. 1). PTI data for each subsection represented the percentage of entire foot pressure. We defined the forefoot PTI as the sum of toe $(\mathrm{A}+\mathrm{B})$ and forefoot pad $(\mathrm{C}+\mathrm{D}) \mathrm{PTI}$, midfoot PTI as the sum of medial and lateral arch (E+F) PTI, and hindfoot PTI as the sum of medial heel and lateral heel $(\mathrm{G}+\mathrm{H})$ PTI. In addition, medial column PTI was determined as the sum of medial forefoot and medial midfoot $(\mathrm{B}+\mathrm{D}+\mathrm{F})$ PTI. Lateral column PTI was determined as the sum of lateral forefoot and lateral midfoot $(\mathrm{A}+\mathrm{C}+\mathrm{E}) \mathrm{PTI}$. The coronal index, the value obtained by subtracting the lateral column PTI from the medial column PTI, was also measured. A more negative value of the coronal index indicates a greater varus foot deformity, and a more positive value of the coronal index indicates a greater valgus foot deformity.

Improvement of the coronal index from a negative value to zero or a positive value suggests shifting of weightbearing from the lateral column to the medial column [2].

\section{Intervention}

BTX-A, Dysport (Ipsen Biopharm Ltd., Wrexham, UK), was used for treatment of spastic equinovarus foot. The choice of muscles and the dosage of BTX-A for injection was decided by multidisciplinary discussion and clinical evaluation, including the severity of spasticity in the affected muscle, the level of activities of daily living, visual inspection of gait pattern, age, and body weight, and results from evaluation of the pre-FPMS. According to severity of the equinus and varus deformity, BTX-A was injected into the GCM at a dose of 6-12 units/ $\mathrm{kg}$ and/or the TPo at a dose of 4-9 units/kg, respectively. For accurate and safe injection of BTX-A, monitored anesthesia care was administered by pediatric anesthesiologists. Bispectral index from the electroencephalogram, oxygen saturation, electrocardiogram, heart rate, and blood pressure were monitored during the BTX-A injection procedure. Electrical stimulation guidance was used for identification of the target neuromuscular junction. Under these 
conditions, the injection procedure was performed with a 23-gauge needle electrode using a sterile technique. A 23-gauge needle electrode was inserted into the GCM or TPo, and adequate needle electrode placement was confirmed with proper movement of the target muscle by electrical stimulation through the inserted needle electrode. After obtaining final confirmation that the location of the needle tip was extravascular by the negative pressure of the syringe, BTX-A was injected. The MAS score, foot contact pattern, and pressure time integral were used to assess the therapeutic effect of BTX-A injection before and three weeks after injection.

\section{Statistical analysis}

Mann-Whitney U-test was used for statistical assessment of the therapeutic effect of BTX-A according to the muscle group. Spearman correlation was used to determine a significant correlation between the change in MAS score of the injected muscles and the change in PTI of each subsection. Statistical analysis was performed using the Statistical Package for Social Sciences (SPSS) ver. 18.0 (SPSS Inc., Chicago, IL, USA). The adopted level of statistical significance was $\mathrm{p}<0.05$. The inter-rater reliability of the data obtained from the FPMS was measured. The intraclass correlation coefficient value between the two experts was 0.911 , indicating excellent reproducibility.

\section{RESULTS}

A summary of demographic data is shown in Table 1. No significant difference was observed in all variables

Table 1. Demographics of patients $(n=18)$

\begin{tabular}{lc}
\hline & Value \\
\hline Age (yr) & $8.79 \pm 4.09$ \\
\hline Sex (male:female) & $15: 3$ \\
\hline Follow-up duration (day) & $21.25 \pm 3.26$ \\
\hline CP (hemiplegic:diplegic) & $14: 4$ \\
GMFCS (level I:II) & $16: 2$ \\
\hline Injected muscle & \\
$\quad$ Gastrocnemius muscle & 14 \\
\hline Tibialis posterior muscle & 12 \\
\hline
\end{tabular}

Values are presented as mean \pm standard deviation or number.

CP, cerebral palsy; GMFCS, Gross Motor Function Classification System. between groups. None of the patients dropped out of the study and no side effects, including sustained pain, focal muscle weakness, fatigue, paresthesia, somnolence, fever, or skin rash, were reported. All patients showed a markedly improved spastic equinovarus pattern during gait.

The results of MAS and foot contact pattern after injection are shown in Table 2. The MAS score of the GCM in 14 limbs which were injected with BTX-A and the MAS score of the TPo in 12 limbs which were injected with BTX-A showed a significant decrease after injection $(p<0.05)$. For foot contact pattern, contact length and contact width, including forefoot, midfoot, and hindfoot width, were increased after the injection, but statistical significance was not reached (Table 2). However, the PTI showed a significant change after injection (Table 3). Lateral column PTI showed significantly decreased values after BTX-A injection. Coronal index showed a significant increase after BTX-A injection, indicating decrease in the varus component of the foot during gait. Although subsections of the forefoot, midfoot, and hindfoot showed no significant changes, the total change in the forefoot and hindfoot PTI was also significant after BTX-A injection. Lateral forefoot pad section was the only subsection that showed a significant change after injection. For specific correlation, injected muscles were categorized into three groups as follows: GCM+TPo injection group,

Table 2. The change in the MAS score and foot contact pattern between before and after injection of botulinum toxin $\mathrm{A}$

\begin{tabular}{|c|c|c|}
\hline & $\begin{array}{c}\text { Before } \\
\text { injection }\end{array}$ & $\begin{array}{c}\text { After } \\
\text { injection }\end{array}$ \\
\hline \multicolumn{3}{|l|}{ MAS score } \\
\hline GCM in 14 limbs & $3.00(1.50)$ & $1.00(0.13)^{*}$ \\
\hline TPo in 12 limbs & $1.50(0.63)$ & $1.00(1.00)^{*}$ \\
\hline \multicolumn{3}{|c|}{ Foot contact pattern $(\mathrm{cm})$} \\
\hline Contact length & $15.90(5.20)$ & $16.8(5.35)$ \\
\hline \multicolumn{3}{|l|}{ Contact width } \\
\hline Forefoot & $8.40(2.30)$ & $8.80(2.70)$ \\
\hline Midfoot & $4.50(1.05)$ & $4.70(0.95)$ \\
\hline Hindfoot & $4.90(1.15)$ & $5.20(1.65)$ \\
\hline
\end{tabular}

Values are presented as median (interquartile range). MAS, Modified Ashworth Scale; GCM, gastrocnemius muscle; TPo, tibialis posterior muscle.

${ }^{*} \mathrm{p}<0.05$. 

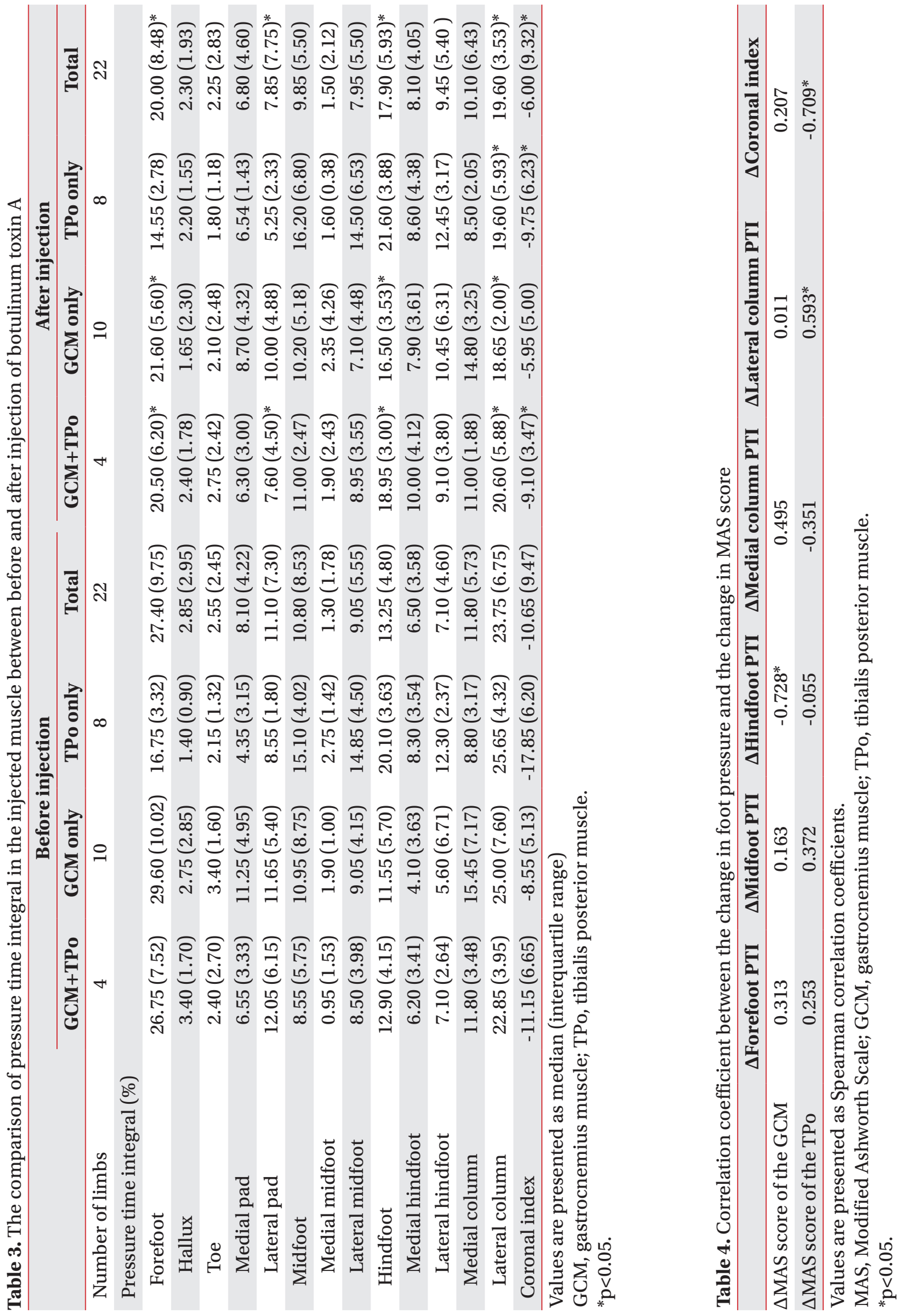
GCM only injection group, and TPo only injection group. Among the 22 limbs, the number of GCM+TPo injected limbs was 4, the number of GCM only injected limbs was 10 , and the number of TPo only injected limbs was 8 . There were meaningful results for the clinical correlation between the injected muscle and PTI. The GCM+TPo and the GCM only injection groups showed a significant decrease in forefoot PTI and a significant increase in hindfoot PTI. Lateral forefoot pad section showed significantly decreased values after injection into the GCM+TPo. The TPo only injection group showed a significantly decreased lateral column PTI. The GCM+TPo and GCM only injection groups also showed a significantly decreased lateral column PTI. For the coronal index, the GCM+TPo and the TPo only injection groups showed significantly increased values after injection.

Results of correlation analysis between the change in MAS score and the change in PTI showed a significant correlation (Table 4). In detail, the change in the MAS score of the GCM showed a significant negative correlation with the change in hindfoot PTI. The change in the MAS score of the TPo showed a significant positive correlation with the change in lateral column PTI and a significant negative correlation with the change in coronal index.

\section{DISCUSSION}

Using the FPMS, we evaluated the change in foot pressure of the spastic equinovarus foot after BTX-A injection. After injection, the result of the FPMS showed a significant increase in hindfoot PTI and coronal index as well as a significant decrease in forefoot PTI, corresponding to the clinical improvement of changes in the MAS score and equinovarus pattern during gait.

Several previous studies have demonstrated the therapeutic effect of BTX-A injection on spasticity in patients with $\mathrm{CP}[8,9,26-31]$. The MAS has been most widely used for assessment of the therapeutic effect of BTX-A. The current study showed results corresponding with those of previous studies, which reported a significant decrease in MAS score after injection. In addition, we used the FPMS for detailed evaluation of the quantitative therapeutic effect of BTX-A injection. One of the parameters of the FPMS, PTI, showed a significant difference in pressure distribution in a specific area of the foot after injection.
PTI data have been used in several previous studies for evaluation of foot deformity [1,2,32-34]. Falso et al. [32], who investigated the therapeutic effect of BTX-A injection in five diplegic and five hemiplegic CP patients, reported that hindfoot PTI showed a significant increase after injection into the GCM, TPo, adductor, and medial hamstring muscles. Another study on PTI by Park et al. [1] also reported significantly increased heel PTI and significantly decreased lateral forefoot PTI after heel cord lengthening surgery in 17 cases of spastic $\mathrm{CP}$ with equinovarus foot. Similarly, our results showed a significant change in PTI. Patients in the GCM only injection group showed a significant decrease in forefoot PTI and a significant increase in hindfoot PTI. It is known that spasticity of the GCM is mainly related with the equinus posture leading to limitation of ankle dorsiflexion and prolonged initial contact time with the forefoot. Improvement of ankle dorsiflexion may enable initial contact with the hindfoot and decrease forefoot PTI. Moreover in this study, the change in the MAS score of the GCM showed a significant negative correlation with the change in hindfoot PTI. These significant changes corresponded with the clinical change in the equinus gait pattern. On the other hand, the GCM+TPo injection group showed a significant change in lateral column PTI as well as in forefoot and hindfoot PTI. The TPo acts mainly in ankle inversion [25]. Thus, spasticity of the TPo can lead to varus foot deformity. Bowen et al. [33] described that equinovarus deformity showed increased PTI and early initiation of contact pressure on lateral forefoot and midfoot. In this study, lateral column PTI showed a significant decrease after BTX-A injection in the GCM+TPo and TPo only injection groups, along with clinical improvement of varus deformity. The results of correlation between the change in lateral column PTI and change in the MAS score of the TPo also showed a significant positive correlation. These findings indicate that reduced spasticity of the TPo is related with decrease in lateral column PTI. Lateral column PTI also showed a significant decrease in the GCM only group after injection. This finding was may be due to a decrease in forefoot PTI. Because the lateral column was composed of second, third, fourth, and fifth toes, lateral forefoot pad, and lateral arch, a decrease in values of these subsection data may cause a decrease in lateral column PTI.

The coronal index, which is determined by the discrep- 
ancy in PTI between the medial and lateral columns, is used to evaluate foot deformities $[1,2]$. In a previous study, Chang et al. [2] reported high correlation of coronal index with clinical characteristics of varus and valgus foot and recommended the FPMS as the primary evaluation tool for measuring the severity of varus or valgus foot deformity. Our patients who received injection into the GCM+TPo and the TPo only also showed a remarkable improvement of varus deformity and the corresponding significant change in coronal index. In addition, a significant correlation was observed between the change in coronal index and the change in MAS score of the TPo. These findings imply that the varus deformity was improved after BTX-A injection into the TPo, which is mainly related to the varus posture, and this was as a result of decreased spasticity of the TPo and shift in the weightbearing pattern from the lateral column to the medial column. For the medial and lateral columns, medial column PTI also decreased after BTX-A injection although it was not significant. It may be possible that this result was caused by the characteristics of medial or lateral column PTI; the medial column PTI was defined as the sum of medial forefoot and medial midfoot PTI, and the lateral column PTI was defined as the sum of lateral forefoot and lateral midfoot PTI. Therefore, the increase in hindfoot PTI was not reflected in medial and lateral column PTI, and a significant decrease in forefoot PTI may be the influential factor on the results of medial and lateral column PTI. As a result, both medial and lateral column PTI showed a tendency to decrease regardless of the muscle injected.

Foot contact pattern, another parameter of the FPMS, has been used in several previous studies on orthopedic corrective surgery for foot deformity $[1,17,23,35]$. These studies reported meaningful correlation between the change in foot contact pattern and radiographic measurements, or considerable change in foot contact area after orthopedic surgery. Our results showed increased contact length and contact width in all subsections; however, these results were not significant. These insignificant results may be related to the difference in therapeutic effect of injection and surgery. In addition, use of the BTX-A injection procedure, which is performed in a specific area of the muscle, may not result in a definite change in total contact length or total contact width.

Gait analysis has also been used for assessment of the therapeutic effect of BTX-A. Some previous studies have reported a significant change in parameters of gait analysis after BTX-A injection $[8,36]$. On the other hand, in some other studies, although patients showed clinical improvement, gait analysis after BTX-A injection did not show any significant result $[37,38]$. Using gait analysis, it is difficult to evaluate the interval change in coronal plane at the foot level. Compared with gait analysis, the FPMS can be a useful evaluation tool to quantify and assess the interval change in the foot in the coronal plane after BTX-A injection.

In conclusion, we demonstrated a statistically significant change in foot pressure distribution after BTX-A injection using the FPMS. A significant change in PTI and a significant correlation between the change in PTI and the change in the MAS score were observed, revealing the quantitative therapeutic effect on the equinus and varus deformity. To the best of our knowledge, this is first report showing a statistical correlation between the change in anteroposterior or mediolateral foot pressure and the change in MAS score of a specific muscle using the FPMS. However, this study has some limitations, including a small number of participants and a short follow-up study period. Additionally, this study was based on clinical needs; hence, it was not possible to uniformly inject into the GCM or TPo. This is another limitation of this study. A future study with a larger number of participants, a long-term follow-up, and a strict process based on the academic concept rather than clinical needs is warranted.

\section{CONFLICT OF INTEREST}

No potential conflict of interest relevant to this article was reported.

\section{ACKNOWLEDGMENTS}

This study was supported by a grant of the Korea Healthcare technology R\&D Project, Ministry for Health, Welfare \& Family Affairs, Republic of Korea (Grant no. A084177).

\section{REFERENCES}

1. Park ES, Kim HW, Park CI, Rha DW, Park CW. Dyna- 
mic foot pressure measurements for assessing foot deformity in persons with spastic cerebral palsy. Arch Phys Med Rehabil 2006;87:703-9.

2. Chang CH, Miller F, Schuyler J. Dynamic pedobarograph in evaluation of varus and valgus foot deformities. J Pediatr Orthop 2002;22:813-8.

3. Tylkowski CM, Horan M, Oeffinger DJ. Outcomes of gastrocnemius-soleus complex lengthening for isolated equinus contracture in children with cerebral palsy. J Pediatr Orthop 2009;29:771-8.

4. Oddy MJ, Brown C, Mistry R, Eastwood DM. Botulinum toxin injection site localization for the tibialis posterior muscle. J Pediatr Orthop B 2006;15:414-7.

5. Mayer NH, Esquenazi A, Childers MK. Common patterns of clinical motor dysfunction. Muscle Nerve Suppl 1997;6:S21-35.

6. Molteni F, Bertoni M, Caimmi M. Equinovarus foot in upper motor neuron syndrome. In: Esquenazi A, editor. Gait analysis. Philadelphia: Hanley \& Belfus; 2002. p. 263-86.

7. Bollens B, Gustin T, Stoquart G, Detrembleur C, Lejeune T, Deltombe T. A randomized controlled trial of selective neurotomy versus botulinum toxin for spastic equinovarus foot after stroke. Neurorehabil Neural Repair 2013;27:695-703.

8. Park CI, Park ES, Shin JC, Kim SW, Kim DY, Ahn JG. Effects of botulinum toxin A therapy on gastrocnemius in spastic cerebral palsied children. J Korean Acad Rehabil Med 1999;23:504-15.

9. Koman LA, Mooney JF 3rd, Smith BP, Walker F, Leon JM. Botulinum toxin type A neuromuscular blockade in the treatment of lower extremity spasticity in cerebral palsy: a randomized, double-blind, placebocontrolled trial. BOTOX Study Group. J Pediatr Orthop 2000;20:108-15.

10. Riad J, Coleman S, Henley J, Miller F. Reliability of pediobarographs for paediatric foot deformity. J Child Orthop 2007;1:307-12.

11. Armstrong DG, Lavery LA. Plantar pressures are higher in diabetic patients following partial foot amputation. Ostomy Wound Manag 1998;44:30-2, 34, 36 passim.

12. Armstrong DG, Peters EJ, Athanasiou KA, Lavery LA. Is there a critical level of plantar foot pressure to identify patients at risk for neuropathic foot ulceration? J Foot Ankle Surg 1998;37:303-7.
13. Holmes GB Jr, Timmerman L. A quantitative assessment of the effect of metatarsal pads on plantar pressures. Foot Ankle 1990;11:141-5.

14. Lavery LA, Armstrong DG, Wunderlich RP, Tredwell J, Boulton AJ. Predictive value of foot pressure assessment as part of a population-based diabetes disease management program. Diabetes Care 2003;26:106973.

15. Minns RJ, Craxford AD. Pressure under the forefoot in rheumatoid arthritis: a comparison of static and dynamic methods of assessment. Clin Orthop Relat Res 1984;187:235-42.

16. Singh N, Armstrong DG, Lipsky BA. Preventing foot ulcers in patients with diabetes. JAMA 2005;293:21728.

17. Oeffinger DJ, Pectol RW Jr, Tylkowski CM. Foot pressure and radiographic outcome measures of lateral column lengthening for pes planovalgus deformity. Gait Posture 2000;12:189-95.

18. Liggio FJ, Kruse R. Split tibialis posterior tendon transfer with concomitant distal tibial derotational osteotomy in children with cerebral palsy. J Pediatr Orthop 2001;21:95-101.

19. Park ES, Rha DW, Choi JE, Park CW, Chung HI. The changes of foot pressure distribution in spastic cerebral palsy with equinus deformity following corrective surgery. J Korean Acad Rehabil Med 2005;29:507-12.

20. Park ES, Park CI, Kim JY, Park JW, Kim EJ. Foot pressure distribution and path of center of pressure (COP) of foot during ambulation in the children with spastic cerebral palsy. J Korean Acad Rehabil Med 2002;26:127-32.

21. Chang $\mathrm{CH}$, Albarracin JP, Lipton GE, Miller F. Longterm follow-up of surgery for equinovarus foot deformity in children with cerebral palsy. J Pediatr Orthop 2002;22:792-9.

22. Huber H, Dutoit M. Dynamic foot-pressure measurement in the assessment of operatively treated clubfeet. J Bone Joint Surg Am 2004;86A:1203-10.

23. Metaxiotis D, Accles W, Pappas A, Doederlein L. Dynamic pedobarography (DPB) in operative management of cavovarus foot deformity. Foot Ankle Int 2000;21:935-47.

24. Boulay C, Jacquemier M, Pomero V, Castanier E, Authier G, Chabrol B, et al. Dynamic EMG of peroneus longus in hemiplegic children with equinovarus. Ann 
Phys Rehabil Med 2014;57:185-92.

25. Ben-Shabat E, Palit M, Fini NA, Brooks CT, Winter A, Holland AE. Intra- and interrater reliability of the Modified Tardieu Scale for the assessment of lower limb spasticity in adults with neurologic injuries. Arch Phys Med Rehabil 2013;94:2494-501.

26. Papavasiliou AS, Nikaina I, Bouros P, Rizou I, Filiopoulos C. Botulinum toxin treatment in upper limb spasticity: treatment consistency. Eur J Paediatr Neurol 2012;16:237-42.

27. Baker R, Jasinski M, Maciag-Tymecka I, MichalowskaMrozek J, Bonikowski M, Carr L, et al. Botulinum toxin treatment of spasticity in diplegic cerebral palsy: a randomized, double-blind, placebo-controlled, doseranging study. Dev Med Child Neurol 2002;44:666-75.

28. Colovic H, Dimitrijevic L, Stankovic I, Nikolic D, Radovic-Janosevic D. Estimation of botulinum toxin type A efficacy on spasticity and functional outcome in children with spastic cerebral palsy. Biomed Pap Med Fac Univ Palacky Olomouc Czech Repub 2012;156:41-7.

29. Degelaen M, de Borre L, Kerckhofs E, de Meirleir L, Buyl R, Cheron G, et al. Influence of botulinum toxin therapy on postural control and lower limb intersegmental coordination in children with spastic cerebral palsy. Toxins (Basel) 2013;5:93-105.

30. Bakheit AM, Severa S, Cosgrove A, Morton R, Roussounis SH, Doderlein L, et al. Safety profile and efficacy of botulinum toxin A (Dysport) in children with muscle spasticity. Dev Med Child Neurol 2001;43:234-8.

31. Lee SJ, Sung IY, Jang DH, Yi JH, Lee JH, Ryu JS. The effect and complication of botulinum toxin type A injec- tion with serial casting for the treatment of spastic equinus foot. Ann Rehabil Med 2011;35:344-53.

32. Falso M, Fiaschi A, Manganotti P. Pedobarometric evaluation of equinus foot disorder after injection of botulinum toxin A in children with cerebral palsy: a pilot study. Dev Med Child Neurol 2005;47:396-402.

33. Bowen TR, Miller F, Castagno P, Richards J, Lipton G. A method of dynamic foot-pressure measurement for the evaluation of pediatric orthopaedic foot deformities. J Pediatr Orthop 1998;18:789-93.

34. Chang CH, Albarracin JP, Lipton GE, Miller F. Longterm follow-up of surgery for equinovarus foot deformity in children with cerebral palsy. J Pediatr Orthop 2002;22:792-9.

35. Davitt JS, MacWilliams BA, Armstrong PF. Plantar pressure and radiographic changes after distal calcaneal lengthening in children and adolescents. J Pediatr Orthop 2001;21:70-5.

36. Sutherland DH, Kaufman KR, Wyatt MP, Chambers HG, Mubarak SJ. Double-blind study of botulinum A toxin injections into the gastrocnemius muscle in patients with cerebral palsy. Gait Posture 1999;10:1-9.

37. Koog YH, Min BI. Effects of botulinum toxin A on calf muscles in children with cerebral palsy: a systematic review. Clin Rehabil 2010;24:685-700.

38. Bottos M, Benedetti MG, Salucci P, Gasparroni V, Giannini S. Botulinum toxin with and without casting in ambulant children with spastic diplegia: a clinical and functional assessment. Dev Med Child Neurol 2003; 45:758-62. 